\title{
Effect Of Addition Of Dexmedetomidine To Ropivacaine In Ultrasound Guided Transverse Abdominis Plexus Block On Postoperative Pain In Open Appendicectomy
} Kamalakar Karampudi ${ }^{1}$, Kavya Waghray ${ }^{2}$

1. Professor, Department of Anesthesiology, Prathima Institute of Medical Sciences, Karimnagar

2. Resident, Department of Anesthesiology, Prathima Institute of Medical Sciences, Karimnagar

Date of submission:17/11/2020

Date of Review: 5/2/2021

Date of Acceptance : 26/4/2021

Corresponding Author: Dr. Kamalakar karampudi, Department of Anesthesia, Prathima Institute of Medical Sciences, Nagnur Road, Karimnagar Telangana.

\section{DOI:10.47799/pimr.0901.13}

\section{ABSTRACT}

INTRODUCTION

Post operative pain is the most common complaint in post anaesthesia care units (PACU). Lower abdominal surgeries are associated with severe post-operative pain and inadequate post-operative analgesia leads to many complications. Multimodality approach is used to treat postoperative pain. Transversus abdominus plane (TAP) block is a peripheral nerve block of the trunk that provides analgesia between T10 to L1 dermatomes with a single injection which was first described by A.N. Rafi ${ }^{1}$ in 2001 . TAP Block is indicated in any lower abdominal surgeries. Local anaesthetic is deposited in the fascial sheath between the internal oblique and transverse abdominis muscle using either the blind or the ultrasound guided technique. Adjuvants are frequently used in regional analgesia for rapid onset to improve the quality and prolong the duration of block. Alpha 2 agonist dexmedetomidine speeds the onset of block and prolongs duration of analgesia.

Aim : To assess whether addition of dexmedetomidine to ropivacaine may bring some improvements to the analgesic efficacy and quality of TAP blocks in patients undergoing appendicectomy surgeries.

\section{MATERIALS AND METHODS}

30 Patients belonging to ASA Class I or II scheduled for appendicectomy were selected for the study .

The patients were randomized into two groups:

Group $\mathbf{R}$ - receiving plain ropivacaine $(2 \mathrm{ml}$ of normal saline and $20 \mathrm{ml} 0.2 \%$ ropivacaine)

Group R+D - receiving ropivacaine with dexmedetomidine $(0.5$ $\mathrm{mcg} / \mathrm{kg}$ of dexmedetomidine dissolved in $2 \mathrm{ml}$ of normal saline and $20 \mathrm{ml} 0.2 \%$ ropivacaine). At the end of surgery, USG guided transversus abdominus plane block was performed.
Results

Addition of dexmedetomidine to ropivacine in TAP block lower pain scores after $3 \mathrm{hrs}$ in postoperative period and the same trend of analgesia continued for the first 24 hours. It confers better post operative analgesia with less analgesic requirement and adverse effects.

\section{Conclusion:}

The addition of dexmedetomidine to ropivacaine in TAP block confers better pain control and decreases the total dose of analgesics post-operatively without any major side-effects. Keywords:

Appendicectomy, Dexmededetomidine, Posto perative Analgesia, Ropivacaine, Transverse Abdominal plain block, Ultrasound Guided

\section{Introduction}

Pain at surgical site is the most common complaint in post anaesthesia care units (PACU). It is a leading cause for patient dissatisfaction and discomfort in the postoperative period. It can also lead to increased length of hospital stay.Lower abdominal surgeries are associated with severe post-operative pain. Inadequate post-operative analgesia leads to many complications and multimodality approach involving the use of opioids, NSAIDS is frequently sought. Peripheral nerve blocks are frequently performed as part of the multimodal approach for postoperative analgesia.Regional anesthesia with peripheral nerve blocks is paramount in postoperative analgesia where the adverse effects associated with opioids and other analgesics as well as those associated with epidural catheter placement can be avoided.

Transversus abdominus plane (TAP) block is a peripheral nerve block of the trunk that provides analgesia 
between T10 to L1 dermatomes with a single injection. It was first described by A.N. Rafi ${ }^{1}$ in 2001 in his article titled "Abdominal field block -a new approach via lumbar triangle ". Four peripheral nerves, namely (i) subcostal; (ii) ilioinguinal;(iii) iliohypogastric and (iv)genitofemoral supply the lower abdomen. The first three of the above-mentioned courses through between the transversus abdominis and internal oblique muscle. It is these nerves that are targeted through the various approaches to obtain analgesia at the lower abdominal surgical plane. TAP Block is indicated in any lower abdominal surgeries including hernia repair, appendicectomy, caesarian deliveries, abdominal hysterectomies etc .The primary indication of this block is for postoperative analgesia in lower abdominal surgeries, it has however been used successfully as solo anaesthetic technique for inguinal hernia repair and appendectomy as well. It is of particular use in patients in whom epidural analgesia is contraindicated. It is performed at the triangle of petit, formed by the iliac crest; latissimus dorsi and external oblique muscle .It is located at the mid axillary line inferior to the lower costal margin and superior to the iliac crest. The local anaesthetic is deposited in the fascial sheath between the internal oblique and transverse abdominis muscle. This block is performed by using either the blind or the ultrasound guided technique. For the blind technique the two-pop approach is used where a blunt needle is passed at the level of lumbar triangle of petite, between the lower costal margin and iliac crest. The two pops are felt when the nerve pierces through the external oblique and Internal oblique muscles, respectively. The drug is deposited deep to the internal oblique muscle after the second pop is felt. This however is associated with possible complications like bowel injury, intrahepatic or intraperitoneal injections.

The use of ultrasonography for performing TAP block has decreased the complications associated with it including bowel injury, accidental intraperitoneal or intra hepatic deposition of local anaesthetic solution, and is now the preferred technique for performing this block. In this a linear probe is used with the patient in supine position, the probe is placed at the midaxillary line between the costal margin and iliac crest, where the three muscles can be well differentiated. Using the in plane technique the drug is deposited between the layers of internal oblique and tranversus abdominus muscles.

Adjutants are frequently used in regional analgesia techniques to speed the onset, improve the quality and prolong the duration of block. Alpha 2 agonists such as clonidine, dexmedetomidine speeds the onset of block and prolongs duration of analgesia. Dexmedetomidine is an alpha 2 agonist with eight times more affinity to alpha 2 receptors than clonidine.

\section{Keywords:}

Appendicectomy, Dexmededetomidine, Posto perative Analgesia, Ropivacaine, Transverse Abdominal plain block, Ultrasound Guided

\section{OBJECTIVE}

The aim is to assess whether addition of dexmedeto midine to ropivacaine may bring some improvements to the analgesic efficacy of TAP blocks in patients undergoing appendicectomy surgeries and to compare the side effects of adding dexmedetomidine to ropivacaine.

\section{MATERIALS AND METHODS}

30 Patients belonging to ASA Class I or II patients and scheduled for appendicectomy were selected for the study from February 2018 to August 2018.

The patients were randomized into two groups:

Group $\mathbf{R}$ - receiving plain ropivacaine $(2 \mathrm{ml}$ of normal saline and $20 \mathrm{ml} 0.2 \%$ ropivacaine)

Group R+D - receiving ropivacaine with dexmedetomidine.

$(0.5 \mathrm{mcg} / \mathrm{kg}$ of dexmedetomidine dissolved in $2 \mathrm{ml}$ of normal saline and $20 \mathrm{ml} 0.2 \%$ ropivacaine)

The patients in the two groups were compared using age, sex, body mass index. Post-operative pain at different intervals was scaled using VAS (visual analogue scale). A metric scale with the markings from $0-10 \mathrm{cms}$ was used. 0 indicating no pain and 10 the worst possible pain felt. Incidence of postoperative headache, nausea, and vomiting and other side effects was recorded. Need for use of opioids and other analgesics in postoperative period were assessed. Inclusion criteria were Patients between ages of 15- 40 yrs and Patients who fit under ASA class 1 and class 2 undergoing appendicectomy. The exclusion criteria were Patients with a history of cardiac, respiratory, renal or hepatic disease, Local infection at the site of block, Psychological disorders, Allergy to study medications and Coagulation disorders.

\section{PROCEDURE}

A Total of 30 patients were equally allocated into two groups of those receiving ropivacaine (group $R$ ) and a combination of ropivacaine and dexmedetomidine (group $R+D$ ) randomly. All demographic data was collected prior to performing the surgery. At the end of surgery, using all standard monitoring techniques a, transversus abdominus plane block was performed. Under all aseptic precaution, with the patient in supine position, abdomen was cleaned and draped. A linear probe was placed transversely between the costal margin and iliac crest at the midaxillary line. A $22 \mathrm{G}$ blunt tipped block needle was used and an in plane ultrasound guided block was performed. Drug was deposited in between the fascial layers of internal oblique and transversus abdominis muscle. Immediate post procedure period the patient was monitored 


\section{Karampudi et al}

for development of any complications associated with the block. The patients were shifted into postoperative care unit, and were monitored. Postoperative pain scores were calculated with the help of visual analogue scale, for its ease of access and better patient acceptability. Pain score were awarded as follows 0 indicated no pain, whereas a score of 10 was given for worst pain possible and recordings were taken at $0,1,3,6,12,18$ and 24 hours post operatively. Patients were also examined for signs of any procedure related complications. The need for additional analgesic use and the time for the first dose of analgesic used was noted in both the groups.Injection tramadol was used as the additional analgesic of choice and the total dosage of drug used in the first 24 hours post surgery was calculated. Development of side effects like nausea, vomiting, headache, dryness of mouth, fall in heart rate, hypotension and sedation in post operative period were also noted. The two groups were then compared for any statistical significant difference.

Figure 1: Procedure for the block

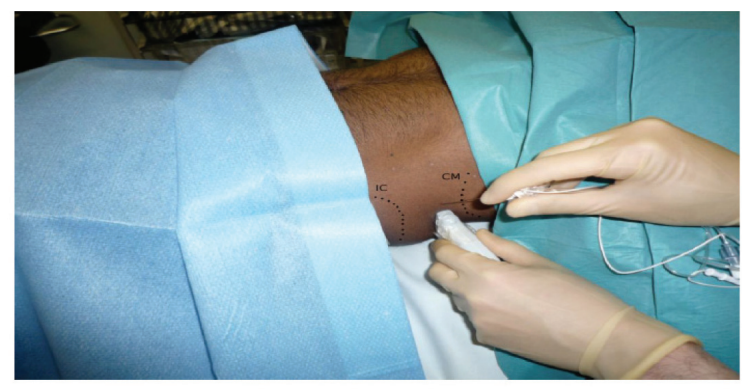

The image produced shows (from above downwards) skin, subcutaneous tissue, fat, external oblique, intern

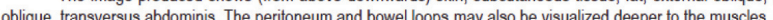

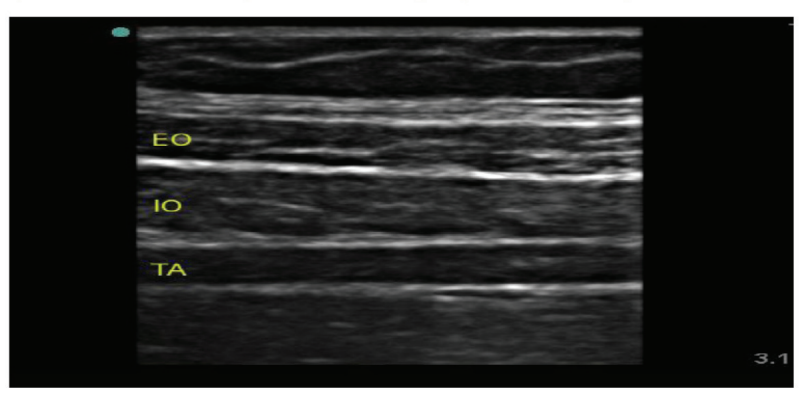

RESULTS-

The two groups were compared in parameters such as age, weight, BMI. The mean age among the two groups was 26.6 and 27.6 respectively. The mean body weight was found to be 56.3 in group $R$ and 55.69 in group $R+D$. the BMI between the two groups was also comparable and no statistical difference with respect to these above mentioned parameters was seen.

The patients were observed postoperatively for pain score in postoperative period at various intervals using the visual analogue scale (VAS). The use of additional analgesic in postoperative period and the time for first dose of the additional analgesic given was noted. www. pimr.org.in

We found that the group receiving ropivacaine with dexmedetomidine had lower pain scores after $3 \mathrm{hrs}$ in postoperative period and the same trend of analgesia continued for the first 24 hours. The need for use of additional analgesics was felt approximately after 90 mins postoperatively in group $R$ as compared to almost $3 \mathrm{hrs}$ in group $\mathrm{R}+\mathrm{D}$. The $\mathrm{p}$ value was calculated and a statistical significance in duration of analgesia from end of surgery $(p=0.024)$ to first need for additional analgesia( $p<0.0001)$ was seen between the two study groups.

The total dosage of opioids (tramadol) given in postoperative period was also calculated and was $140 \mathrm{mg}$ in group $\mathrm{R}$ and only $100 \mathrm{mg}$ in group R+D. A smaller dosage of opioids was required in the first $24 \mathrm{hrs}$ in the group receiving additional dexmedetomidine. Significant difference was noted with a $p$ value of $<0.0003$ between the groups.

The use of dexmedetomidine is often associated with bradycardia, hypotension and sedation especially at higher doses; none of which were seen in our study. Other postoperative side effects associated with opioid usage like nausea and vomiting were observed in both the groups but were however not significant.

Table 1: Demographic variables of the patients

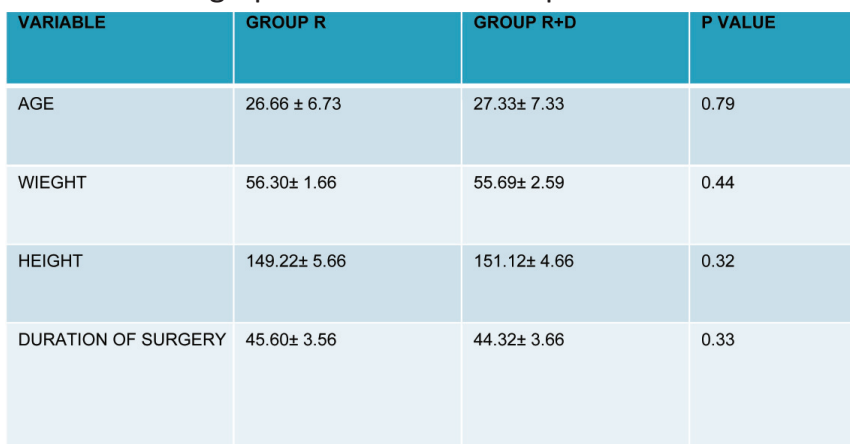

Table 2: Visual Analog scale for both groups

\begin{tabular}{|l|l|l|l|}
\hline DURATION in Hrs & GROUP R & GROUP R+D & P- VALUE \\
\hline 0 & - & - & - \\
\hline 1 & - & - & - \\
\hline 3 & $2.92 \pm 2.22$ & $1.16 \pm 1.8$ & 0.024 \\
\hline 6 & $2.82 \pm 1.66$ & $1.55 \pm 1.50$ & 0.031 \\
\hline 12 & $3.62 \pm 2.12$ & $2.22 \pm 1.22$ & 0.034 \\
\hline 18 & $3.55 \pm 1.56$ & $2.02 \pm 1.62$ & 0.013 \\
\hline 24 & $3.56 \pm 2.46$ & $2.63 \pm 3.85$ & 0.437 \\
\hline
\end{tabular}

Table 3: Side effects associated with the medications

\begin{tabular}{|l|c|c|}
\hline & Group R & Group R+D \\
\hline Nausea & 2 & 1 \\
\hline Headache & 1 & 1 \\
\hline Vomiting & 2 & 1 \\
\hline
\end{tabular}




\begin{tabular}{|l|c|c|}
\hline Dryness of mouth & - & - \\
\hline Bradycardia & - & - \\
\hline Hypotension & - & - \\
\hline Sedation & - & - \\
\hline
\end{tabular}

Discussion

Pain is the most common complaint in post operative period and a source of discomfort and dissatisfaction to the patients in the post anesthesia care unit. Inadequate pain control has often seen to lead to not only development of further complications in the recovery period but also to prolonged stay in the postoperative care units. Post operative pain control is therefore cornerstone in anesthetic practice. Multiple modalities for pain control are now available, from analgesic drugs such as NSAID's, opioids etc. to regional anaesthesia techniques like epidural analgesia and peripheral nerve blocks. Multiple techniques have been introduced for analgesia by peripheral nerve blocks which are easy to perform and avoid the specific complications associated with either use of analgesic drugs (eg-nausea and vomiting for opiods) and epidural analgesia. Transverses abdominis plane block is one such peripheral nerve block which can be performed in post operative period for lower abdominal surgeries (egappendecectomy) with minimal post procedural complications as was also seen in study conducted by McDonnell and O'Donnell2. It was first described by A.N. Rafi ${ }^{1}$ in 2001 in his article titled "Abdominal field block -a new approach via lumbar triangle ". The use of this block has been advocated in multiple studies conducted over the past decade and emphasis has been laid on the use of ultrasound guided block for better efficacy and fewer complications that are associated with this technique. Lissauer J,Maucuso $\mathrm{K}{ }^{3}$ have also supported the better analgesic efficacy of TAP block and its opioid sparing effect in their study.In our study we have used the ultrasound guided technique to perform the block for its fewer rate of complications and better analgesic efficacy as was also advocated by in the study conducted by Hsiao-Chien Tsai et al ${ }^{4}$.

Adjuvants are added to local anaesthetic agents to prolong the duration of block and improve quality of block performed. Epinephrine, opioids, alpha 2 agonists,etc. are frequently used in clinical practice. Dexmedetomidine an alpha 2 agonist with higher affinity for alpha 2 receptors than clonidine has been used in regional anesthesia for both neuraxial as well as peripheral blocks. This study was undertaken to establish the analgesic efficacy of addition of dexmedetomidine to ropivacaine for TAP block in post operative pain management of patients undergoing appendicectomy surgery. Patients belonging to ASA grade I and II were taken and patients were divided into two equal numbered groups receiving only ropivacaine (group $\mathrm{R}$ ) and a combination of ropivacaine and dexmedetomidine(group $R+D$ ) in preset dilutions. The patients were observed postoperatively for pain score in postoperative period at various intervals using the visual analogue scale (VAS). Readings were taken at 0 , $1,3,6,12,18$ and 24 hours post operatively. The use of additional analgesic in postoperative period and the time for first dose of the additional analgesic given was noted. We found that the group receiving ropivacaine with dexmedetomidine had lower pain scores after $3 \mathrm{hrs}$ in postoperative period and the same trend of analgesia continued for the first 24 hours. The need for use of additional analgesics was felt approximately after 90 minutes postoperatively in group $\mathrm{R}$ as compared to almost $3 \mathrm{hrs}$ in group R+D. A significant difference in duration from end of surgery to first need for additional analgesia was seen between the two groups. The total dosage of opioids (tramadol) given in postoperative period was also calculated and was $140 \mathrm{mg}$ in group R and only $100 \mathrm{mg}$ in group R+D. A smaller dosage of opioids was required in the first $24 \mathrm{hrs}$ in the group receiving additional dexmedetomidine. These findings are consistent with those of the meta-analysis conducted by Sun Q, Liu S., et al 5and a separate study by Jadon A,Jain P., and others ${ }^{6}$ both of which found that dexmedetomidine as adjuvant showed lower postoperative pain score and reduced opioid consumption rate. Similar results were reported by Bansal and Garg ${ }^{7}$ in their study. Raof RA,El metainy SA ${ }^{8}$ conducted a study with bupivacaine and dexmedetomidine and their findings are comparable to ours.

The use of dexmedetomidine is often associated with bradycardia, hypotension and sedation especially at higher doses.Almarakbi, W. A., \& Kaki, A. M. ${ }^{9,10}$ in their study had reported bradycardia without any hemodynamic compromise in the first 60 minutes of administration of block with dexmedetomidine as adjuvant, no such finding was seen in our study. Other postoperative side effects associated with opioid usage like nausea and vomiting were observed in both our study groups but where not significant.

Our study concluded a better analgesic efficacy of ropivacaine with dexmedetomidine as compared to ropivacaine alone with respect to duration of analgesia in post operative period. It also showed the decrease in total dosage of opioids used in the 24 hour postoperative period in study population with dexmedetomidine as adjuvant to ropivacaine.

\section{CONCLUSION:}

- Addition of dexmedetomidine to ropivacaine in TAP block provides prolonged post-operative analgesia and better pain control.

- Need for rescue analgesic doses was less when dexmedetomidine was added to ropivacaine 
- The addition of dexmedetomidine to ropivacaine in TAP block confers better pain control and decreases the total dose of analgesics post-operatively without any major sideeffects.

\section{REFERENCES}

1. Rafi AN. Abdominal field block: a new approach via the lumbar triangle. Anaesthesia. 2001 Oct; 56(10):1024-6. doi: 10.1046/j.1365-2044.2001.02279-40.x. PMID: 11576144.

2. McDonnell, John G., MB, FCARCSI*+; O'Donnell, Brian, MB, FCARCSIt; Curley, Gerard, MB*; Heffernan, Anne, MB, FCARCSIt; Power, Camillus, MD, FCARCSIt; Laffey, John G., MD, MA, FCARCSI* $\ddagger$ The Analgesic Efficacy of Transversus Abdominis Plane Block After Abdominal Surgery: A Prospective Randomized Controlled Trial, Anesthesia \& Analgesia: January 2007 - Volume 104 - Issue 1 - p 193-197doi: 10.1213/01.ane.0000250223.49963.0f

3. Lissauer J, Mancuso K, Merritt C, Prabhakar A, Kaye AD, Urman RD. Evolution of the transversus abdominis plane block and its role in postoperative analgesia. Best Pract Res Clin Anaesthesiol. 2014 Jun;28(2):117-26. doi: 10.1016/j.bpa.2014.04.001. Epub 2014 May 9. PMID: 24993433.

4. Tsai, H. C., Yoshida, T., Chuang, T. Y., Yang, S. F., Chang, C. C., Yao, H. Y., Tai, Y. T., Lin, J. A., \& Chen, K. Y. (2017). Transversus Abdominis Plane Block: An Updated Review of Anatomy and Techniques. BioMed research international, 2017, 8284363. https://doi.org/10.1155/ $2017 / 8284363$

5. Sun, Q., Liu, S., Wu, H., Ma, H., Liu, W., Fang, M., Liu, K., \& Pan, Z. (2019). Dexmedetomidine as an Adjuvant to Local Anesthetics in Transversus Abdominis Plane Block: A Systematic Review and Meta-analysis. The Clinical journal of pain, 35(4), 375-384. https://doi.org/10.1097/ AJP.0000000000000671

6. Jadon, A., Jain, P., Chakraborty, S., Motaka, M., Parida, S. S., Sinha, N., Agrawal, A., \& Pati, A. K. (2018). Role of ultrasound guided transversus abdominis plane block as a component of multimodal analgesic regimen for lower segment caesarean section: a randomized double blind clinical study. BMC anesthesiology, 18(1), 53. https:// doi.org/10.1186/s12871-018-0512-x

7. Bansal, Prannal MD*; Garg, Shashank MD, MS+ Effect of Adding Dexmedetomidine to Local Anesthetic Agents for Transversus Abdominis Plane Block, The Clinical Journal of Pain: October 2019 - Volume 35 - Issue 10 - p 844-854 doi: 10.1097/AJP.0000000000000742

8. Raof, R. A., El Metainy, S. A., Alia, D. A., \& Wahab, M. A. (2017). Dexmedetomidine decreases the required amount of bupivacaine for ultrasound-guided transversus abdominis plane block in pediatrics patients: a randomized study. Journal of clinical anesthesia, 37, 5560. https://doi.org/10.1016/j.jclinane.2016.10.041

9. Almarakbi, W. A., \& Kaki, A. M. (2014). Addition of dexmedetomidine to bupivacaine in transversus abdominis plane block potentiates post-operative pain relief among abdominal hysterectomy patients: A prospective randomized controlled trial. Saudi journal of anaesthesia, 8(2), 161-166. https://doi.org/10.4103/ 1658-354X.130683

10. Nandanwankar N,Abdullah M F. Evaluation of TransversusAbdominis Plane (Tap) Block with Inj.Bupivacaine $0.25 \%$ For Post-Operative Analgesia In Inguinal Hernia Repair Surgery.Perspectives in Medical Research 2019; 7(3): 30-37

How to cite this article : Karampudi K,Waghray K. Effect Of Addition Of Dexmedetomidine To Ropivacaine In Ultrasound Guided Transverse Abdominis Plexus Block On Postoperative Pain In Open Appendicectomy Perspectives in Medical Research 2021; 9 (1):64-68

DOI:10.47799/pimr.0901.13

Sources of Support: Nil, Conflict of interest: None declared 